\title{
Compatibility of Staining Protocols for Bone Tissue with Raman Imaging
}

\author{
M. D. Morris, ${ }^{1}$ N. J. Crane, ${ }^{1}$ L. E. Gomez, ${ }^{2}$ M. A. Ignelzi $\mathbf{J r}^{2}$ \\ ${ }^{1}$ Department of Chemistry, University of Michigan, 930 N. University Avenue, Ann Arbor, MI 48109-1055, USA \\ ${ }^{2}$ Department of Orthodontics and Pediatric Dentistry, University of Michigan School of Dentistry, Ann Arbor, MI, USA
}

Received: 17 February 2003 / Accepted: 6 May 2003 / Online publication: 20 November 2003

\begin{abstract}
We report the use of Raman microscopy to image mouse calvaria stained with hematoxylin, eosin and toluidine blue. Raman imaging of stained specimens allows for direct correlation of histological and spectral information. A line-focus $785 \mathrm{~nm}$ laser imaging system with specialized near-infrared (NIR) microscope objectives and $\mathrm{CCD}$ detector were used to collect approximately $100 \times 450 \mu \mathrm{m}$ Raman images. Principal components analysis, a multivariate analysis technique, was used to determine whether the histological stains cause spectral interference (band shifts or intensity changes) or result in thermal damage to the examined tissue. Image analysis revealed factors for tissue components and the embedding medium, glycol methacrylate, only. Thus, Raman imaging proved to be compatible with histological stains such as hematoxylin, eosin and toluidine blue.
\end{abstract}

Key words: Raman spectroscopy - Histological staining - Mineralized tissue - Bone - H\&E - Toluidine blue

Raman microspectroscopy and Raman imaging are increasingly used to provide chemical structure information on tissue specimens. While spectroscopy provides valuable information, biomedical scientists usually wish to link the information to histological analysis [1-4]. Depending upon the staining protocols employed, histology may provide morphological information, identification of broad classes of molecules such as proteins and nucleic acids, or may provide highly specific identification by means of labeled antibodies or other molecules that bind uniquely to one protein. Histological analysis can also identity regions of a specimen that merit further investigation by spectroscopy. Correlative histological/Raman imaging may provide information that is beyond what is available by either methodology. For such reasons it is important to

Correspondence to: M. D. Morris; E-mail: mdmorris@ umich.edu understand the compatibility of histological and spectroscopic protocols and to identify any sources of incompatibility.

In this study we investigated the compatibility of hematoxylin and eosin (H\&E) staining and toluidine blue staining with near-infrared (NIR) Raman imaging of fetal day 18.5 and postnatal day 21 mice calvaria, or flat bones that comprise the skull. Raman microspectroscopy and imaging provide molecular structure information with light microscopy spatial resolution [5]. Raman spectra are obtained by focusing a laser on the specimen. While low laser power is usually used, the possibility of thermal damage is always present. For Raman microscopy it is advantageous to use line-focused lasers that result in low power at any point along the line. This configuration simultaneously reduces the likelihood of laser damage and provides a quick survey of the tissue along the line.

We evaluated H\&E staining because it is among the most widely used staining techniques. Hematoxylin is an acidophilic stain derived from the dye hematein. Staining with hematoxylin results in nuclei and basophilic cytoplasm colored blue. Eosin, a basophilic dye, is the common counterstain for hematoxylin. Acidophilic cytoplasm and connective tissue turn varying shades of pink to red with eosin. Together, H\&E clearly contrast the different tissue components. We have also investigated the effects of toluidine blue, which is often used alone, but can be substituted for hematoxylin. Toluidine blue also stains nuclei and basophilic cytoplasm blue [6].

Raman spectroscopy of tissue specimens is generally excited with NIR lasers. NIR lasers are preferred to the more common visible wavelength lasers because NIR light does not excite native fluorescence from most fluorophores in tissues, although fluorescence is excited over most of the range of visible wavelengths (400-700 $\mathrm{nm})$. Red stains, such as eosin, absorb at blue-green to ultraviolet wavelengths $(250-500 \mathrm{~nm})$ but not at red or near-infrared wavelengths. However, blue stains, such as hematoxylin, absorb at red wavelengths $(650-700 \mathrm{~nm})$ and their absorbance spectra may extend into the 
near-infrared $(>700 \mathrm{~nm})$. Thus, blue stains cannot be considered innocuous for Raman spectroscopy until their absorption spectra have been examined for absorbance at the laser excitation wavelength being used.

We have previously shown that Raman image contrast of bone tissue shows both morphology and chemistry [7-10]. Using multivariate data analysis (principal component analysis, PCA) even minor components of the specimen can be identified [1] and in some cases can be imaged. Serial sections can be used to compare spectroscopic results with histological results. One section is examined by spectroscopy and an adjacent section is subjected to histological analysis. It is accepted that there are small histological variations between serial sections, which makes it somewhat inaccurate to compare sections. A more objective approach of correlative studies between spectroscopy and histopathology would be to examine spectroscopically the same sections that are examined histologically. In many previous studies, spectroscopic sample preparation consists of snap freezing tissue and sectioning with a cryostat [11-14]. The examined tissue is unfixed and unstained [14-16]. The sections subjected to histological analysis are fixed, typically in formalin which retains tissue rigidity and prevents bacterial growth, then embedded and stained with $H \& E$.

Correlative studies depend on the staining being compatible with the spectroscopic technique. There are two main concerns with staining and Raman microscopy. The first is whether or not the stain will interfere spectroscopically. If there is Raman signal from the stain, it must be distinguishable from other tissue components by PCA. The second concern is whether the stain will absorb at the laser wavelength and generate heat which in turn will destroy the specimen. Previous studies show that basic fuchsin does not interfere spectroscopically nor does it cause thermal damage [9]. Here we determine the compatibility of Raman microscopy and tissues stained with $H \& E$ and toluidine blue.

\section{Materials and Methods}

\section{Experimental Subjects}

B6CBA $F_{1} / \mathrm{J}$ wild-type mice (The Jackson Laboratory, Bar Harbor, ME) were harvested at fetal day 18.5 and postnatal day 21 . We used two ages of mice to determine compatability of staining and Raman imaging with delicate tissue (fetal day 18.5 ) and more mature tissue with more mineral (postnatal day 21). The calvaria, flat bones from the topmost portion of the skull, were excised and rinsed with a phosphate-buffered saline solution at $\mathrm{pH}$ 7.2. Specimens were then fixed in either formalin or ethanol and stored at $4^{\circ} \mathrm{C}$. Prior to embedding with glycol methacrylate (Technovit 1800 GMA: Energy Beam Sciences, Agawam, MA), calvaria were dissected and placed in a 50/50 mixture of infiltrating solution and absolute ethanol for 2 hours and then in infiltrating solution overnight. Infil- trated specimens were oriented in the polyethylene mold tray, submerged with embedding solution and topped with a plastic holder for sectioning. Specimens were left to cure overnight at $4^{\circ} \mathrm{C}$.

Embedded specimens were sectioned at $20 \mu \mathrm{m}$ with glass knives in a JB-4 microtome (Energy Beam Sciences) and then mounted onto a glass microscope slide (Probe-On Plus, Fisher Scientific, Pittsburgh, PA) and allowed to air dry. H\&E staining procedures for GMA-embedded tissue were followed for a portion of the sections [17]. Other sections were used to study modified staining protocols in which submersion time in hematoxylin solution was varied ( $30 \mathrm{sec}, 1 \mathrm{~min}, 2 \mathrm{~min}$, $5 \mathrm{~min}$ and $7 \mathrm{~min}$ ). Other sections were stained with toluidine blue and eosin, again following the GMA-embedded tissue protocol.

\section{Raman Analysis}

The Raman imaging system has been described previously [7]. Briefly, about $160 \mathrm{~mW}$ of line-focused laser light from a 785 run diode laser (Invictus, Kaiser Optical Systems, Inc., Ann Arbor, MI) is delivered to the sample via epi-illumination with a microscope (BH-2; Olympus, Inc., Melville, NY) equipped with a NIR-optimized $20 \times 0.75 \mathrm{NA}$ objective (Fluar Series, Carl Zeiss, Inc., Thornwood, NY). Raman scatter collected through the objective is focused into an axial-transmissive spectrograph (HoloSpec f/1.8i, Kaiser Optical Systems, Inc., Ann Arbor, MI) operated at $3-4 \mathrm{~cm}^{-1}$ resolution $(25 \mu \mathrm{m}$ entrance slit). The dispersed signal is collected by a $1024 \times 128$ pixel charge coupled device camera (DU401-BR-DD; Andor Technology, South Windsor, CT) operated at $-75^{\circ} \mathrm{C}$. To obtain an image, a stepper-motor controlled $x-y$ translation microscope stage (NEAT, Salem, NH) moves the sample in 1.5 $\mu \mathrm{m}$ steps for the desired number of frames, providing $3 \mu \mathrm{m}$ spatial resolution, with 3 min acquisitions per frame. In most cases 150 frames were collected, to yield a $126 \times 150$ pixel image containing information from all 18,900 Raman spectra. Brightfield images were collected by a color video camera (TMC-7; Pulnix, Sunnyvale, CA) mounted on the microscope frame. All absorbance spectra were collected with a Shimadzu 2101 Scanning UV-Visible Spectrophotometer. All scans were performed from $550-800 \mathrm{~nm}$, with $0.1 \mathrm{~nm}$ slit width, $0.5 \mathrm{~nm}$ sampling intervals and a fast scan speed.

\section{Factor Analysis}

Factor analysis [19], which has been discussed previously [10, 18], was performed with Matlab 5.3 (MathWorks, Inc., Natick, MA), using both manufacturer-supplied routines as well as in-house developed routines. The entire set of 18,900 Raman spectra that comprise an image is treated as a matrix of 18,900 rows (spectra) and 1024 columns (Raman shifts). The principal components, which are the eigenvectors of this matrix, are calculated and then sorted by their magnitudes, called eigenvalues. The standard procedure for calculating eigenvectors is called "singular value decomposition" and is included in Matlab. The eigenvectors are a mathematically orthogonal coordinate system (i.e., extensions of the familiar 3-dimensional Cartesian coordinate system) from which the original data set can be calculated. The number of eigenvectors found is equal to the number of Raman spectra. However, almost all of the non-random variation in the image data set can be described by the eigenvectors with the largest magnitudes. Usually between four to eight eigenvectors are sufficient. The remaining eigenvectors describe the random noise in the data set and are not used in the data analysis. The criterion for eigenvector retention is that addition of the next eigenvector describes no more than $0.1 \%$ of the variation in the data set. The intensities of these eigenvectors are called scores.

Principal components are mathematically orthogonal and so may contain both positive and negative bands. They are sums and differences of the underlying Raman spectra. To convert 


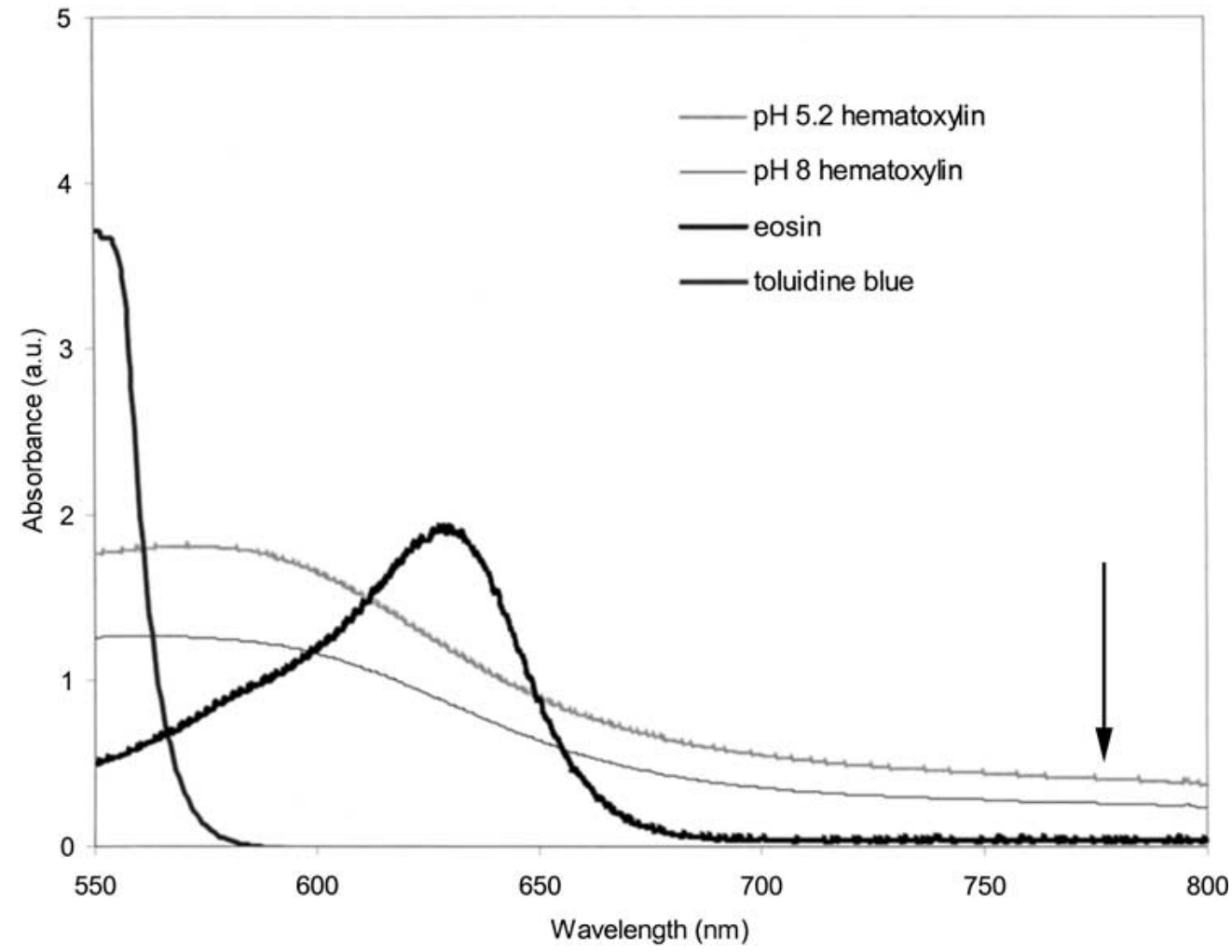

Fig. 1. Absorbance spectra of $0.01 \%$ hematoxylin solution at $\mathrm{pH} 5.2$ and $8,0.01 \%$ eosin solution and $0.01 \%$ toluidine blue solution. The arrow points to absorbances at the wavelength of excitation, approximately $785 \mathrm{~nm}$.

the retained principal components to Raman spectra, weighted sums and differences are calculated, with the weighting subject to two constraints. The first constraint is that at each Raman shift the calculated weighted sums and differences be nonnegative, as are the Raman spectra. A second constraint is that at each point the reconstructed Raman spectra must add up to the original intensity. These constraints are sufficient to guarantee that the reconstructed spectra, called factors, will be directly proportional to the real Raman spectra. The relative intensity (score) images will be positive and will be maps of the spatial distribution and relative amounts of indicating where and how much of the components are present in the specimen.

\section{Results}

Absorption of Histological Stains

In order to determine if eosin, hematoxylin and toluidine blue would cause sufficient heating upon laser exposure to cause tissue damage, the absorption at the laser wavelength was determined. Figure 1 shows the absorption spectra of eosin, hematoxylin and toluidine blue from $550 \mathrm{~nm}$ to $800 \mathrm{~nm}$. Although the spectra were obtained in solutions of the stains for experimental convenience, the spectra of the solid phase stains are similar. Eosin shows no detectable absorbance at 785 $\mathrm{nm}$, as would be expected for a red stain. Toluidine blue absorbs weakly at $785 \mathrm{~nm}$ (0.04 from a $0.01 \%$ solution). Hematoxylin absorbs more strongly at $785 \mathrm{~nm}(0.4$ at $\mathrm{pH} 5.2$ and 0.3 at $\mathrm{pH} 8$, from a $0.01 \%$ solution).

\section{Light Microscopy of Stained Specimens}

Brightfield images of mouse calvaria embedded in GMA and stained according to the protocols are shown (Fig. 2). Raman images of regions of these specimens are presented and discussed below. The image of a control section of GMA (no tissue), part of which is stained with eosin, is shown in Figure 2a. The image of a second control, a section of GMA-embedded and unstained calvarial tissue from a 21-day-old wild-type mouse is shown in Figure 2b. Figure $2 \mathrm{c}$ shows the same section after staining with eosin. Note, the holes in $2 \mathrm{~b}$ and $2 \mathrm{c}$ are a result of the embedding process, not tissue damage by the laser. Consistent with the data presented in Figure 1, no thermal damage was detectable after Raman imaging was performed on these sections. Figure $2 \mathrm{~d}$ shows a section of fetal day 18.5 calvarial tissue stained with eosin and toluidine blue. The presence of toluidine blue did not result in any detectable thermal damage, even though it is weakly absorbing at $785 \mathrm{~nm}$.

Several H\&E protocols resulted in enough bound hematoxylin to cause thermal damage under $785 \mathrm{~nm}$ laser illumination. Figure 2e shows a section of calvarial tissue from a 21-day-old wild-type mouse stained by an $H \& E$ protocol that consisted of requiring $15 \mathrm{~min}$ immersion in hematoxylin. Note the laser damage to the upper right hand corner of the specimen. Visible damage, burning of the tissue, often accompanied by noticeable 


\section{a}
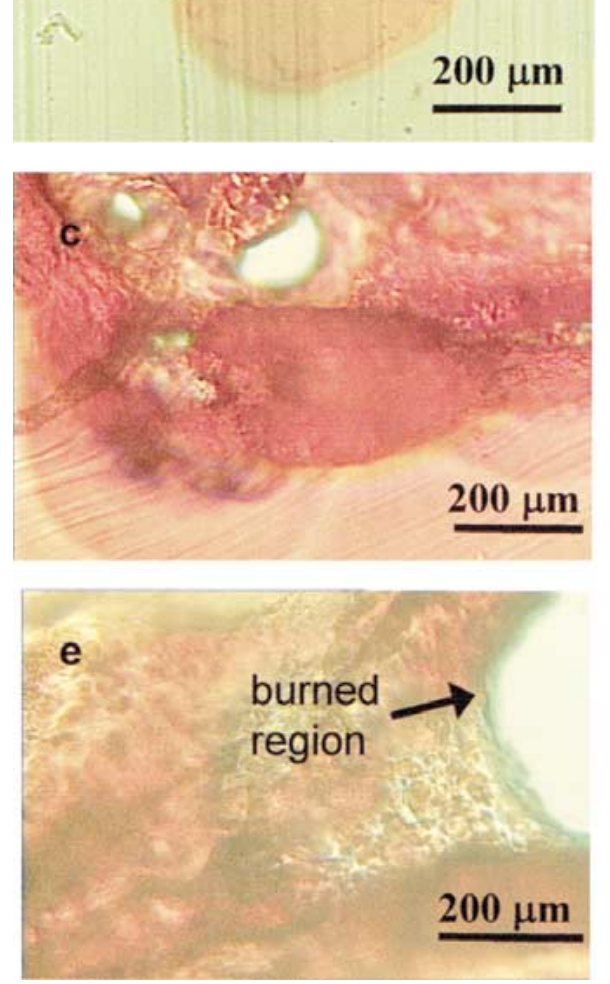

Fig. 2. Brightfield images of sections studied with Raman imaging. Sections were of the following: (a) GMA partially stained with eosin, (b) postnatal day 21 mouse calvaria embedded in GMA and unstained, (c) postnatal day 21 mouse calvaria embedded in GMA and stained with eosin, (d) fetal

odor, occurred as soon as the laser was incident on the specimen. A video image was obtained less than 1 second later. Not surprisingly then, protocols requiring longer immersion in hematoxylin also resulted in damage.

Hematoxylin immersion was reduced in a series of steps to find conditions that resulted in good contrast in reflectance imaging with no visible or spectroscopically detectable thermal damage. Fetal tissue was used for testing these protocols because it is more easily damaged than mature tissue. Immersion times of 7, 5, 2, 1 and $0.5 \mathrm{~min}$ were examined. Sections immersed in hematoxylin for 2 , 5 , and 7 min burned immediately. Specimens immersed in hematoxylin for $1 \mathrm{~min}$ sustained $1.5 \mathrm{~min}$ of laser exposure before visible damage was observed. Immersion in hematoxylin solution for $0.5 \mathrm{~min}$ resulted in no thermal damage, even after more than $5 \mathrm{~min}$ of exposure to the laser. There was little difference in contrast obtained after the 0.5 -minute immersion and the longer H\&E staining protocols, as shown by comparison (Fig. 2e,f). day 18.5 mouse calvaria embedded in GMA and stained with toluidine blue and eosin, (e) fetal day 18.5 mouse calvaria embedded in GMA and stained by standard H\&E protocol, (f) fetal day 18.5 mouse calvaria embedded in GMA and stained by modified H\&E protocol.

\section{Raman Imaging of GMA Stained with Alcoholic Eosin}

Figure $2 \mathrm{a}$ shows a reflectance brightfield image of a GMA section (no tissue) that was used to test for spectroscopic interference from eosin (only part of the section was stained with eosin). Figure 3a shows the only Raman factor recovered from the Raman imaging experiment and $3 \mathrm{~b}$ shows the corresponding score image.

In Figure $3 \mathrm{a}$ only the spectral bands between 420 $\mathrm{cm}^{-1}$ and $1045 \mathrm{~cm}^{-1}$ are shown in order to bring out detail that emphasizes the similarities between corresponding factors obtained here and in the staining protocol experiments described below. Spectra were obtained over the $420-1800 \mathrm{~cm}^{-1}$ range and contain additional Raman bands that are not shown. For consistency, the Raman images throughout the paper have similar dimensions to the image of Figure $3 \mathrm{~b}$, approximately $100 \mu \mathrm{m} \times 450 \mu \mathrm{m}$.

If the eosin stain produces a Raman spectrum that is detectable, there will be eosin bands in the Raman 


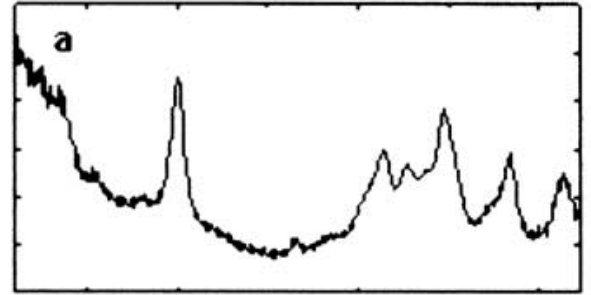

$\begin{array}{llllll}500 & 600 & 700 & 800 & 900 & 1000\end{array}$ Raman Shift $\left(\mathrm{cm}^{-1}\right)$
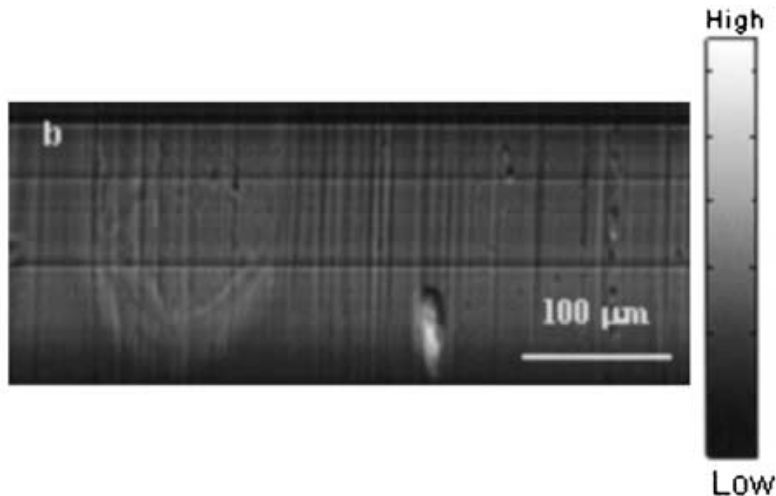

Fig. 3. Raman factor for a section of GMA partially stained with eosin (a) and the corresponding score image (b). Note only GMA is detectable. The section examined was $20 \mu \mathrm{m}$ thick, mounted on a glass microscope slide. Laser power incident to the sample was $185 \mathrm{~mW}$.

spectra from this part of the section but not from the unstained part. The extra bands should be resolvable by factor analysis even if they are not readily visible upon inspection. However, as indicated in Figure 3, the eosin is not visible spectroscopically. Only one factor containing Raman bands is obtained. This factor is representative of the typical GMA spectrum, with signature bands at $600 \mathrm{~cm}^{-1}$ and $968 \mathrm{~cm}^{-1}$. The score (intensity) is uniform across the section, with no difference between the stained and unstained regions. Only striations from the microtome glass knife and one surface irregularity mar the otherwise flat surface. We conclude that if eosin spectra are present, they must contribute much less than $1 \%$ to the total observed Raman intensity.

\section{Raman Imaging of Calvaria Embedded in GMA and Unstained}

To determine whether spectral interference would result from the application of histological stains, we first resolved the factors of the GMA embedded tissue without staining. Figure $2 b$ is the reflectance brightfield image of the region of a section of calvaria from a 21-day postnatal mouse. The specimen here, calvaria from a 21-day postnatal mouse, is embedded in GMA and unstained. It serves as a control for comparison with images from stained specimens. Three non-background Raman spectrum factors are observed. The factors and the corresponding score images are shown in Figure 4.

The GMA factor (Fig. 4a) is identical to the GMA factor shown in Figure 3a. Bone matrix (Fig. 4c) and bone mineral (Fig. 4e) factors are also obtained. These are identical to factors that we have previously reported for similar mouse calvaria specimens [7]. The corresponding score images are Figures 4b (GMA), 4d (bone matrix) and $4 \mathrm{f}$ (bone mineral). There is some overlap between the GMA image and the bone mineral and matrix images, but it is not great. Though there may be some infiltration into the tissue, the GMA shows greatest intensity where bone is not present in the sec- tion. It is clear that factor analysis adequately separates the components of the specimen, which is composed of GMA (Fig. 4a), bone matrix (Fig. 4c) and bone mineral (Fig. 4e).

\section{Raman Imaging of Calvaria Embedded in GMA and Stained}

In Figure 5 Raman score images from a selected region of eosin-stained tissue specimen of Figure $2 \mathrm{c}$ are shown. Three non-background factors that contain Raman spectral information for the specimen were found. The remaining factors contained only background luminescence and were not included. The three Raman factors correspond to GMA (Fig. 5a), bone mineral (Fig. 5c) and bone matrix (Fig. 5e). As found for the unstained GMA-embedded calvaria, the GMA is found (Fig. 5b) mostly where the bone mineral (Fig. 5d) is absent, although it does fill small depressions in the tissue. In comparing the bone mineral and matrix (Fig. 5f) score images there is a relatively uniform distribution of mineral on the matrix, as would be expected of a small region of mature tissue such as this 21-day-old mouse. There was no factor found for the eosin. The stain is probably present in a sufficiently enough thin layer that it cannot be detected spectroscopically.

A fetal day 18.5 mouse calvarial section stained with toluidine blue and eosin is shown in Figure 6. As discussed above, the tissue was chosen because it is more easily damaged than mature tissue. There are two nonbackground factors, GMA (Fig. 6a) and bone (Fig. 6c). The corresponding score images are shown as Fig. $6 \mathrm{~b}$ and $6 \mathrm{~d}$. Although mineralization is expected and present in this tissue, it is not as extensive as in the postnatal day 21 tissue. The bone factor appears at lower signal/ noise ratio than in the more mature tissue specimens used in the Figs. 4 and 5. No Raman factors are found for toluidine blue because the stain is present in a thin layer. 
for thermal compatibility with Raman imaging. From the absorption spectra, one would expect that eosin staining would not result in thermal damage, even to delicate tissue, but that damage could result from absorption by either of the blue stains, hematoxylin or toluidine blue. Stained sections should always be inspected for thermal damage after exposure to the laser light. Additionally, even if there is no visual evidence of thermal damage, Raman spectra from control unstained sections and adjacent stained sections should be compared for subtle differences. In particular, Raman shift frequencies and band intensities change when a protein is denatured thermally or chemically. Any changes must be presumed to be evidence of thermal damage to the tissue, unless further study demonstrates otherwise. Similarly, chemical alterations as a result of staining could result in Raman factors different from the Raman factors of unaffected tissue. However, the stain is usually a superficial layer that affects a small fraction of the specimen. All band frequency and intensity changes in this study are attributed to sample-to-sample variations.

General conclusions can be drawn about the compatibility of Raman microspectroscopy or imaging and histological staining. There are no compatibility problems if a stain does not absorb laser radiation. If nearinfrared lasers are used, one would not expect thermal damage resulting from red stains such as eosin or basic fuchsin. Blue stains cannot be assumed to be innocuous. With the $785 \mathrm{~nm}$ laser used in these experiments, toluidine blue does not cause any thermal damage because it is only weakly absorbing at this wavelength. Hematoxylin absorbs $785 \mathrm{~nm}$ more strongly but by reducing the hematoxylin immersion time to $0.5 \mathrm{~min}$, thermal damage was eliminated with only slight loss in contrast. It may be necessary to modify standard staining protocols when using a laser line that lies within the absorption spectrum of a given stain.

A second concern when using Raman imaging is whether or not the stain(s) will spectroscopically interfere with the Raman signal of tissue components. It is clear that stains, i.e., eosin, hematoxylin and toluidine blue, do not interfere with Raman imaging as evidenced by lack of Raman factors in Figures 3, 4, 5, 6, 7. Immersion in these stains does not interfere with resolution of tissue components by factor analysis. Comparisons with unstained specimens revealed that the factors representing bone mineral and bone matrix are unchanged. Close examination and comparison of Figures 4 (unstained mouse calvaria embedded in GMA) and 5 (mouse calvaria embedded in GMA and stained with eosin) reveal no spectral differences.

NIR Raman spectroscopy is favored for tissue specimens because tissue fluorescence is only weakly excited. Line focusing reduces local power density more than $100 \times$ compared to point focusing of the same laser beam $[20,21]$. This advantage is obtained even if the goal of an experiment is not acquisition of a complete two-dimensional spectroscopic image of a specimen. While line focusing reduces the likelihood of damage to delicate specimens, it cannot eliminate the problem completely. Therefore, it is prudent to test for thermal damage before attempting Raman microspectroscopy or imaging of tissue that has been subjected to blue staining.

Acknowledgments. Research was supported by NIH DE11530 (to M.A.I), NIH AR47969 (to M.D.M.), U.S. Army Research Office DAMD17-01-1-0809 (to M.D.M.), and University of Michigan Rackham School of Graduate Studies Summer Research Opportunity Program Fellowship (L.E.G.). The authors would like to thank John Baker (University of Michigan) for his assistance with sample preparation.

\section{References}

1. Manoharan R, Shafer K, Perelman L, Wu J, Chen K, Deinum G, Fitzmaurice M, Myles J, Crowe J, Dasari RR, Feld MS (1998) Raman spectroscopy and fluorescence photon migration for breast cancer diagnosis and imaging. Photochem Photobiol 67:15-22

2. Romer TJ, Brennan III JF, Fitzmaurice M, Feldstein ML, Deinum G, Myles J, Kramer JR, Lees RS, Feld MS (1998) Histopathology of human coronary artherosclerosis by quantifying its chemical composition with Raman spectroscopy. Circulation 97:878-885

3. Fitzmaurice M (2000) Principles and pitfalls of diagnostic test development: implications for spectroscopic tissue diagnosis. J Biomed Optics 5:119-130

4. Zonios G, Cothren R, Crawford JM, Fitzmaurice M, Manoharan R, van Dam J, Feld MS Spectral pathology. Ann NY Acad Sci, pp 108-115

5. Carden A, Morris MD (2000) Application of vibrational spectroscopy to the study of mineralized tissues (review). J Biomed Optics 5:259-268

6. Opperman LA, Passarelli RW, Morgan EP, Reintjes M, Ogle RC (1995) Cranial sutures require tissue interactions with dura mater to resist osseous obliteration in vitro. J Bone Miner Res 10:1978-1987

7. Tarnowski CP, Ignelzi MA, Morris MD (2002) Mineralization of developing mouse calvaria as revealed by Raman microspectroscopy. J Bone Miner Res 17:11181126

8. Lin D-L, Tarnowski CP, Zhang J, Dai J, Rohn E, Patel AH, Morris MD, Keller ED (2001) Bone metastatic LNCaP-derivative C4-2B prostate cancer cell line mineralizes in vitro. Prostate 47:212-221

9. Timlin J, Carden A, Morris MD, Rajachar RM, Kohn DH (2000) Raman spectroscopic imaging markers for fatigue-related microdamage in bovine bone. Anal Chem 72:2229-2236

10. Timlin JA, Carden A, Morris MD (1999) Chemical microstructure of cortical bone probed by Raman transects. Appl Spectroscopy 53:1429-1435

11. Bakker Schut TC, Puppels GJ, Kraan YM, Greve J, van der Maas LLJ, Figdor CG (1997) Intracellular cartenoid levels measured by Raman microspectroscopy: comparison of lymphocytes from lung cancer patients and healthy individuals. Int J Cancer 74:20-25

12. Bakker Schut TC, Witjes MJH, Sterenborg HJCM, Speelmen OC, Roodenburg JLN, Marple ET, Bruining HA, Puppels GJ (2000) In vivo detection of dysplastic tissue by Raman spectroscopy. Anal Chem 72:6010-6018

13. Manoharan R, Wang Y, Feld MS (1996) Histochemical analysis of biological tissues using Raman spectroscopy. Spectrochim Acta 52:215-249

14. Buschman HP, Deinum G, Motz JT, Fitzmaurice M, Kramer JR, van der Laarse A, Bruschke AV, Feld MS 
(2001) Raman microspectroscopy of human coronary atherosclerosis: biochemical assessment of cellular and extracellular morphologic structures in situ. Cardiovasc Pathol 10:69-82

15. Deinum G, Rodriguez D, Römer TJ, Fitzmaurice M, Kramer JR, Feld MS (1999) Histological classification of Raman spectra of human coronary artery atherosclerosis using principal component analysis. Appl Spectroscopy 53:938-942

16. Lasch P, Haensch W, Lewis EN, Kidder LH, Naumann D (2002) Characterization of colorectal adenocarcinoma sections by spatially resolved FT-IR microspectroscopy. Appl Spectroscopy 56:1-9

17. Gerrits PO, van Leeuwen MBM (1987) Glycol methacrylate embedding in histotechnology: the hematoxylin- eosin stain as a method for assessing the stability of glycol methacrylate sections. Stain Technol 62:181190

18. Shaver JM, Christensen KA, Pezzuti JA, Morris MD (1998) Structure of dihydrogen phosphate ion aggregates by Raman-monitored serial dilution. Appl Spectroscopy 52:259-264

19. Malinowski ER (2002) Factor analysis in chemistry. John Wiley and Sons, New York

20. Treado PJ, Morris MD (1989) A 1000 points of light - the Hadamard-transform in chemical analysis and instrumentation. Anal Chem 61:723A

21. Zhang D, Hanna JD, Jiang Y, Ben-Amotz D (2001) Influence of laser illumination geometry on the power distribution advantage. Appl Spectroscopy 55:61-65 